\title{
Reparo de desvio peniano em bovinos com implante de ligamento nucal alógeno*
}

\section{Penis deviation repair in cattle with implant of allogenous nuchal ligament}

\author{
Duvaldo Eurides, ${ }^{* *}$ João Luiz Cansanção de Azevedo, ${ }^{* * *}$ Juliana Januzi Roquette, ${ }^{* * *}$ Lucas Vilela Perroni Silva, ${ }^{* * * *}$ \\ Marina Greco Magalhães Guerra de Andrade, ${ }^{* * * *}$ Luiz Augusto de Souza*****
}

\begin{abstract}
Resumo
Sete novilhos mestiços, com idade entre 12 e 18 meses, foram submetidos à remoção do ligamento apical do pênis. Após 60 dias, na presença de fêmeas em estro, verificaram-se desvios ventral e lateral direito do pênis, com incapacidade de cópula. Foi realizada uma incisão na superfície dorsal do pênis e um segmento do ligamento nucal alógeno preservado em glicerina a $98 \%$ foi implantado sobre a túnica albugínea. Decorridos 60 dias, os animais foram observados em monta natural e apresentaram correção do desvio de pênis sem alteração do comportamento sexual. O implante de ligamento nucal alógeno conservado em glicerina a 98\%, implantado sobre a túnica albugínea, promove a correção do desvio ventral lateral e direito de pênis de bovinos, sem alterar a libido.
\end{abstract}

Palavras-chave: infertilidade, túnica albugínea, reprodução, glicerina.

\begin{abstract}
Seven crossbred calfs, aged between 12 and 18 months, were subjected to removal of the apical ligament of the penis. After 60 days, in the presence of females in estrus, was found ventral and right lateral deviation of the penis, with inability to copulate. Was performed an incision on the dorsal surface of the penis and a segment of the allogenous nuchal ligament, preserved in glycerin $98 \%$, was implanted over the tunica albuginea. After 60 days, the animals were observed in natural mating and showed correction of penis deviation without changing sexual behavior. The implantation of allogenous nuchal ligament, preserved in glycerin $98 \%$, implanted on the tunica albuginea, promotes repair of ventral and right lateral deviation of the penis in cattle, without affecting the libido.
\end{abstract}

Keywords: infertility, tunica albuginea, reproduction, glycerin.

\section{Introdução}

Touros de diferentes raças, com idade entre 1,5 e 9 anos, apresentam alta incidência de desvio peniano, sobretudo em animais acima de 2,5 anos de idade (Mihura e Campero, 1995). No entanto, Aiello e Mays (2001) relataram que a maior ocorrência do desvio ocorre de 2 a 11 anos, sendo os mais afetados com idade de três a seis anos, devido à incapacidade de introduzir o pênis e completar a cópula. O desvio de pênis pode ser em espiral (Steffen, 1997), ventral (Walker, 1980), em forma de "S" (Van Camp, 1997). O desvio ventral do pênis acontece por incapacidade do ligamento apical em sustentar a extremidade do pênis durante a ereção (Van Camp, 1997). A extremidade livre do pênis se curva para baixo e impede sua introdução na vagina (Aiello e Mays, 2001). A alteração pode ser congênita ou de caráter hereditário (Mobini e Walker, 1983).

O ligamento apical de bovinos é uma faixa fibrosa longitudinal que se origina na face dorsal da túnica albugínea, distalmente a $7,0 \mathrm{~cm}$ da flexura sigmoide, e se insere na túnica albugínea, nas proximidades do ápice (Schaller, 1999). Em touros da raça Girolanda apresenta, aproximadamente, largura de $0,7 \mathrm{~mm}$ e espessura de 1,9mm (Cardoso et al., 2010). Possui a função de sustentar o pênis reto (Hafez, 1995). Encontra-se separado da túnica albugínea por tecido conjuntivo frouxo, exceto na origem e na inserção (Mobini e Walker, 1983). A remoção do ligamento apical na extremidade livre do pênis causa desvio do pênis ventral à direita (Eurides e Silva, 2002).

São descritas técnicas para corrigir o desvio de pênis, como a fixação com pontos de fio de náilon do ligamento apical à túnica albugínea peniana (Ashdown e Pearson, 1973) e remoção parcial da túnica albugínea e do ligamento apical do pênis com reflexão lateral dessas estruturas (Ashdown e Pearson, 1973). Assim como implantes de fibras de carbono sobre a túnica albugínea (Mobini et al, 1982), borracha de silicone (Eurides et al., 1994), tela de silicone (Boyd e Hanselka, 1972), cartilagem auricular (Eurides et al., 1994), tendão autólogo do músculo

*Recebido em 4 de agosto de 2014 e aceito em 12 de dezembro de 2015.

**Departamento de Cirurgia Veterinária da Faculdade de Medicina Veterinária da Universidade Federal de Uberlândia.

***Graduação em Medicina Veterinária - Faculdade de medicina Veterinária - Universidade Federal de Uberlândia.

****Programa de Pós-graduação em Ciência Veterinárias (Mestrado) - Saúde Animal - Universidade Federal de Uberlândia. Rua Ceará s/n, Bloco 2D, sala 3, Campus Umuarama. Uberlândia, Minas Gerais. Brasil. (34) 9973-0938.

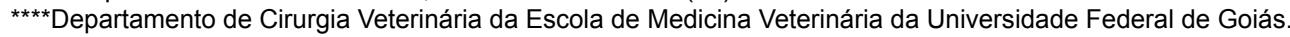

Autor para correspondência: Iperronivet@gmail.com. 
flexor superficial dos dedos (Eurides et al., 2007) e malha de polipropileno e placa de quitosana (Rabelo, 2009).

A glicerina a $98 \%$ tem sido utilizada para preservação de biomaterais por ser antisséptico com amplo espectro de ação e fixador que desidrata o tecido, substituindo a maior parte de água intracelular, sem alterar a concentração iônica das células (Guimarães et al., 2007). O tempo de conservação na glicerina para que o biomateral perca a capacidade de estimular antigenicidade deve ser de ao menos 30 dias (Costa Neto, 2000).

Objetivou-se promover a correção do desvio de pênis de bovinos com implante, sobre a túnica albugínea, de ligamento nucal alógeno conservado em glicerina a $98 \%$ e avaliar a eficácia do implante, quanto à correção do desvio, de forma que o procedimento realizado possa ser utilizado na prática cirúrgica para restabelecimento da ereção peniana.

\section{Material e métodos}

Seguimentos do ligamento nucal alógenos foram lavados, por várias vezes, com solução salina $0,9 \%$, removidos os fragmentos de tecidos moles, coágulos sanguíneos. Foram novamente irrigados submetidos a antissepsia com iodopovidona ${ }^{1}$ tópico a $10 \%$. Removeu-se do ligamento frações de aproximadamente $3,0 \mathrm{~cm}$ de largura, $6,0 \mathrm{~cm}$ de comprimento e $0,3 \mathrm{~cm}$ de espessura e armazenou-se em frascos esterilizados contendo solução de glicerina ${ }^{2}$ a $98 \%$, onde permaneceram submersas e conservadas à temperatura ambiente, por um período mínimo de 60 dias.

O projeto foi aprovado pelo comitê de ética na utilização de animais número 011/12, protocolo registro CEUA/UFU 135/11 para utilização de sete novilhos mestiços com idade entre 12 e 18 meses e peso corporal entre $120 \mathrm{~kg}$ e $160 \mathrm{~kg}$. Os animais, identificados com numeração de 1 a 7 , foram mantidos em jejum hídrico por 12 horas e de alimentos sólidos por 24 horas. Foram sedados com acepromazina ${ }^{3}$ a $1 \%(0,1 \mathrm{mg} / \mathrm{kg}, \mathrm{IM})$ e anestesia regional peridural caudal com $10 \mathrm{ml}$ de lidocaína ${ }^{4}$ a $2 \%$ para facilitar a exposição do pênis. A pele do óstio prepucial foi tricotomizada e submetida à antissepsia com álcool $70 \%$ seguida pela aplicação de iodopovidona tópica. Em seguida praticou-se uma incisão longitudinal na lâmina interna do prepúcio, no terço médio da superfície dorsal da extremidade livre do pênis, a $1,0 \mathrm{~cm}$ da glande e a $1,0 \mathrm{~cm}$ da inserção da lâmina externa do prepúcio e removeu-se o ligamento apical do pênis (Figura 1 - A) para promover desvio ventral e a direita do pênis (Eurides e Silva, 2002). A pele foi aproximada com pontos simples separados com categute cromado $00^{5}$.

\footnotetext{
${ }^{1}$ Riodeine. Rioquímica Indústria Farmacêutica. São José do Rio Preto, SP. Brasil.

${ }^{2}$ Rioquímica Indústria Farmacêutica. São José do Rio Preto, SP. Brasil.

${ }^{3}$ Acepran. Vetnil Indústria e Comércio de Produtos Veterinários Ltda. Louveira, SP. Brasil

${ }^{4}$ Xylestesin. Cristália Produtos Químicos Farmacêuticos Ltda. Itapira, SP. Brasil.

${ }^{5}$ Brasuture. Suturas Cirúrgicas. São Sebastião da Grama, SP. Brasil
}

No pós-operatório, $(\mathrm{PO})$ realizaram-se curativos diários através de lavagens com solução salina $0,9 \%$ e infusão de nitrofurazona ${ }^{6}$ $0,2 \%$ na cavidade prepucial durante três dias, e receberam enrofloxacino ${ }^{7} 10 \%$ (2,5mg/kg/dia) pela via intramuscular durante seis dias consecutivos. Os pontos da lâmina interna do prepúcio foram removidos decorridos 10 dias de PO. Depois de 60 dias de $\mathrm{PO}$ os novilhos foram avaliados na presença de vaca em estro para confirmar a presença do desvio ventral e a direita do pênis (Figura 1 - B).
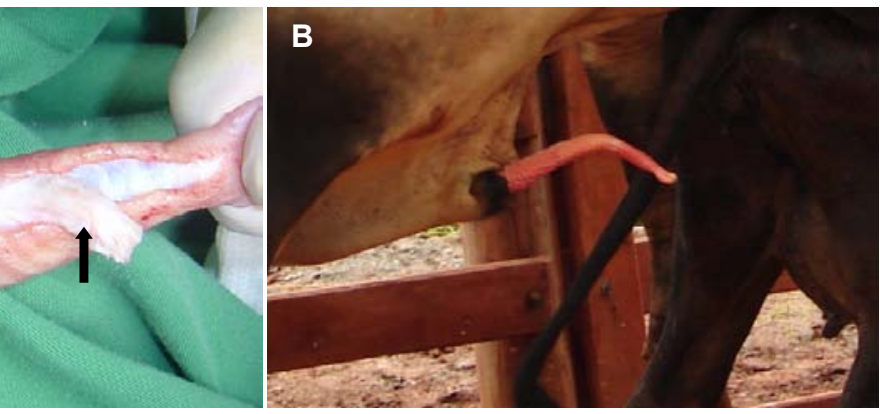

Figura 1: Remoção do ligamento apical na extremidade livre do pênis do bovino 3 (A - seta) e a existência de desvio ventral do pênis decorridos 60 dias de PO no bovino 4(B).

Os animais foram submetidos ao mesmo protocolo préoperatório e anestésico para a cirurgia corretiva do desvio com implante de segmento do ligamento nucal homólogo, retirado de peças frigoríficas e conservado em glicerina a $98 \%$. O implante foi mantido em solução salina a $0,9 \%$ durante 30 minutos para reidratação. Foi praticada uma incisão longitudinal na superfície dorsal do pênis, em média $6,0 \mathrm{~cm}$ de comprimento, iniciada em entorno de $1,0 \mathrm{~cm}$ da extremidade caudal do colo da glande e terminada a $1,0 \mathrm{~cm}$ da inserção da lâmina externa do prepúcio. Semelhante ao comprimento do ligamento apical removido, um segmento do ligamento nucal alógeno de $0,3 \mathrm{~cm}$ de espessura foi implantado sobre a túnica albugínea com fio de algodão $00^{8}$ (Figura 2 - A) e a lâmina interna do prepúcio aproximada com categute cromado $00^{9}$, com pontos simples separados (Figura 2 - B).

Foram realizadas observações clínicas diárias durante 60 dias quanto aos aspectos do local da incisão de pele, abscessos e deiscências da sutura. Realizaram-se os mesmos procedimentos pós-operatórios e decorridos 60 dias de $\mathrm{PO}$ os animais foram avaliados em monta natural para verificar a correção do desvio e a capacidade de realizar cópula. Após o período preestabelecido, os animais foram novamente submetidos aos mesmos procedimentos pré-operatórios, sedação e anestesia no local do implante para colheita de um segmento do implante com a túnica albugínea de aproximadamente $1,0 \times 1,0 \mathrm{~cm}$. As amostras colhidas foram fixadas em solução aquosa de formol a $10 \%$ e para processamento histológicos corados com hematoxilina eosina (HE).

${ }^{6}$ Riocin. Rioquímica Indústria Farmacêutica. São José do Rio Preto, SP. Brasil. ${ }^{7}$ Baytril. Bayer Saúde Animal. São Paulo, SP. Brasil

${ }^{8}$ Brasuture. Suturas Cirúrgicas. São Sebastião da Grama, SP. Brasil

${ }^{9}$ Brasuture. Suturas Cirúrgicas. São Sebastião da Grama, SP. Brasil 


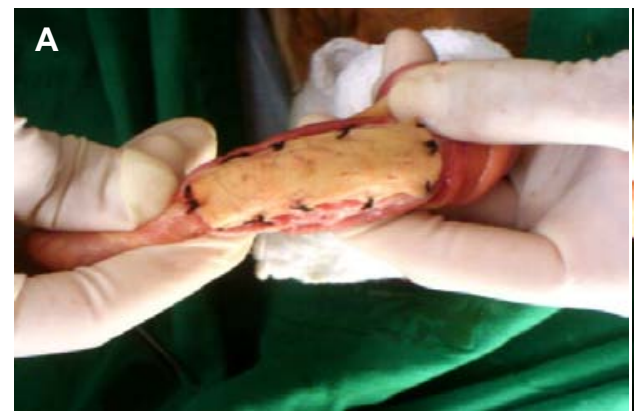

Figura 2: Implante de ligamento nucal alógeno conservado em glicerina sobre a túnica albugínea na extremidade livre do pênis do bovino $3(2-A)$ e aproximação da lâmina interna do prepúcio com pontos simples separados bovino $3(2-B)$.

\section{Resultados e discussão}

Traumatismos penianos em bovinos ocasionam desvios, sobretudo o espiral (Rabelo, 2009), com possibilidade de ruptura da túnica albugínea e do corpo cavernoso (Van Camp, 1997). A remoção do ligamento apical do pênis com implante de um segmento do ligamento nucal alógeno conservado em glicerina, realizada neste experimento, ocasionou adesão uniforme e resistente entre o ligamento e a túnica albugínea, corrigindo a disfunção da fibroarquitetura da túnica albugínea e do corpo cavernoso, o que ocorre nos casos traumáticos destas estruturas (Rabelo, 2009).

Diferentes métodos com resultados favoráveis foram propostos para correção do desvio de pênis em bovinos, como formação de duas faixas do ligamento apical corrigir desvio em espiral (Walker, 1980) e fixação do ligamento à túnica albugínea (Van Camp, 1997). Assim como implante de fáscia lata autóloga (Mobini e Walker, 1983), de cartilagem auricular autóloga (Eurides et al., 2009; tendão autólogo (Eurides et al., 2007), borracha de silicone (Eurides et al., 1994), malha de polipropileno e placa de quitosana (Rabelo, 2009). Neste experimento, o ligamento nucal sobre a túnica albugínea ocasionou a formação de tecido conjuntivo fibroso, com firme aderência fibrosa do ligamento com túnica albugínea, como referido por Eurides et al. (1994) com borracha de silicone e Rabelo (2009), com malha de polipropileno e quitosana. $O$ implante do ligamento nucal e a aderência formada impediu a formação de desvios. O implante somado às aderências fibrosas contribuiu na sustentação ereta do pênis durante a realização da cópula

A remoção de uma seção do ligamento apical na extremidade livre do pênis dos novilhos ocasionou desvio peniano ventral e lateral direito, com incapacidade em realizar a cópula (Eurides e Silva, 2002). A efetividade da técnica na correção do desvio de pênis, observada nesse trabalho, foi devida à consistência do tecido do ligamento nucal homógeno implantado e a sua adesão estável à túnica albugínea.

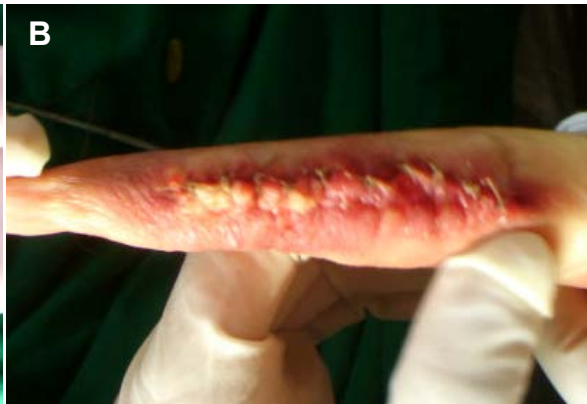

Aos 60 dias de $\mathrm{PO}$, os animais, quando na presença de fêmeas no estro, não mostraram disfunção na ereção e na realização da cópula (Figura 3 ). 0 reparo do desvio peniano reforça a indicação de procedimentos cirúrgicos para a correção desta afecção (Aiello e Mays, 2001).

Neste experimento, o implante de ligamento nucal alógeno proporcionou o retorno da capacidade de sustentação do pênis, desviado pela remoção do ligamento apical da túnica albugínea de sua extremidade livre devido à presença do ligamento e da formação de tecido conjuntivo fibroso envolvendo o implante e a própria túnica.

No exame histopatológico notou-se a presença de processo inflamatório no enxerto que se caracterizou pela formação de tecido de granulação, presença de fibroblastos, grande quantidade de vasos sanguíneos e fibras colágenas neoformadas, ou seja, intensa atividade de fibroplasia (Figura 4). O tecido de granulação se transformou em cicatriz composta de fibroblastos fusiformes, colágeno denso, fragmentos de tecido elástico e componentes da matriz extracelular. O ligamento nucal alógeno implantado serviu como condutor de fibras colágenas.
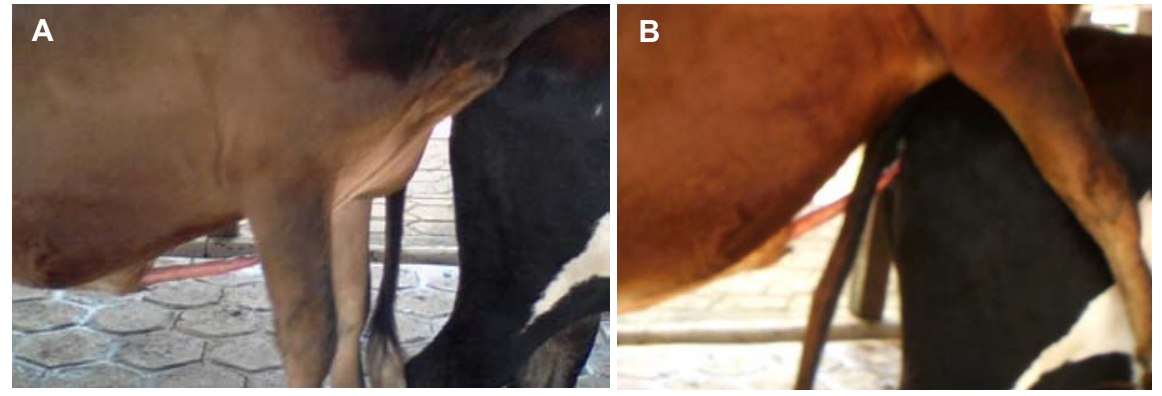

Figura 3: Bovinos 1 e 2 submetidos ao implante de ligamento nucal alógeno sobre a túnica albugínea e avaliados aos 60 dias de PO. Notar a correção do desvio com retorno da capacidade de sustentação ereta do pênis $(A$ e $B)$.
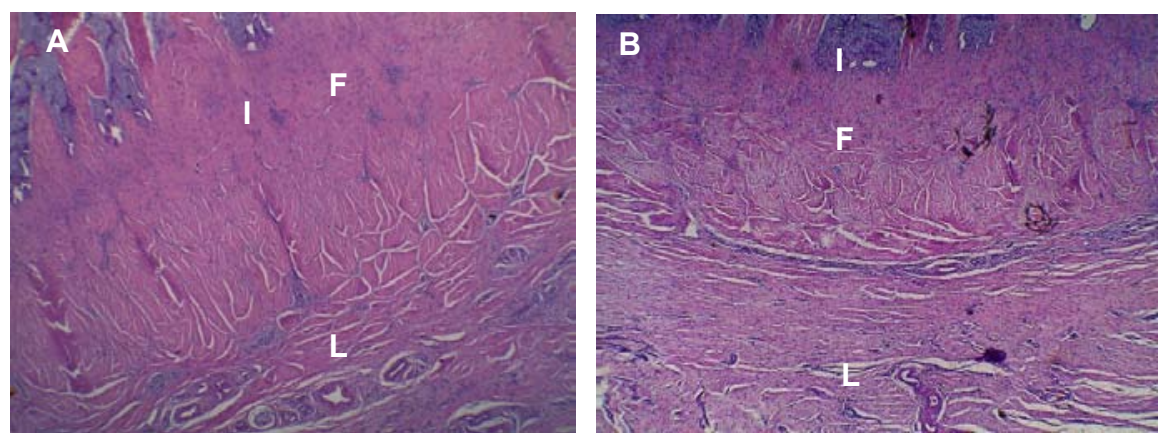

Figura 4: Aspecto histológico da extremidade livre do pênis do bovino 2 com enxerto de segmento de ligamento nucal alógeno aos 60 dias de PO. Notar em A e B, processo inflamatório (I), intensa fibroplasia $(F)$ envolvendo o ligamento nucal (L), HE. 100x.

Quanto ao uso do fio de sutura para reparo de desvio de pênis Boyd e Hanselka (1972), utilizaram o náilon na síntese da tela de dácron na transfixação do ligamento apical à túnica albugínea 
e Walker (1980) o categute para fixar o ligamento em forma de faixas, assim como para aproximar o implante de fibra de carbono (Mobini et al., 1982). Já Ashdown e Pearson (1971) sugeriram a fixação do ligamento à túnica com fio de náilon e Mobini e Walker (1983) fio de ácido poliglicólico para implante de fáscia lata. Entretanto, Eurides et al. (2007) implantaram um segmento de tendão autólogo sobre a túnica albugínea com pontos simples separados de fio algodão, como utilizado neste experimento, por manter o enxerto firmemente fixado à túnica albugínea por um longo período de tempo.

Foram utilizados enxertos alógenos para a correção de desvio de pênis de bovinos, como fáscia lata (Walker, 1980; Mobini e Walker, 1983), cartilagem auricular (Eurides et al., 1994) e tendão músculo flexor superficial dos dedos (Eurides et al., 2007). Não

\section{Referências}

AIELLO, S.E.; MAYS, A. Manual Merk de veterinaria. 10. ed. São Paulo: Roca, 2014. p. 3472.

ASHDOWN, R.R.; PEARSON, H. The functional significance of the dorsal apical ligament the penis. Res. Vet. Sci., v.12, p.183184, 1971.

ASHDOWN, R.R.; PEARSON, N. Studies on "corkscrew" in the bull. Vet. Rec., v. 93, p. 30-35, 1973.

BOYD, C.L.; HANSELKA, D.V. Implantation of the silicone prosthesis for correction of bovine penile deviation. J. Am. Vet. Med. Assoc., v.161, n. 3, p. 275-277, 1972.

CARDOSO, J.R.; EURIDES, D.; MOREIRA, P.C.; MENDONÇA, A.C.; SILVA, L.A.F.; CARNEIRO, F.O.; CRUZ, S.V.S. Morfometria e biometria do ligamento apical do pênis de touros da raça Girolando. Ciência. Rural, v. 40, n. 8, p.1759-1764, 2010.

COSTA NETO, J.N. Tenoplastia experimental do calcâneo em cães, com ligamento nucal de bovino conservado em glicerina a 98\%. Botucatu, 2000. 84 f. Tese (Doutorado em Medicina Veterinária) - Faculdade de Ciências Agrárias e Veterinárias, Universidade Estadual Paulista, Botucatu.

EURIDES, D.; SILVA, M.; COELHO, H.E.; CONTESINI, E.A. Implante de borracha de silicone em pênis de bovinos. Estudo experimental. Ciência. Rural, v. 24, n. 3, p. 545-550, 1994.

EURIDES, D.; SILVA, L.A.F. Métodos de preparo de rufiões bovinos e equinos. Goiânia: Talento, 2002. 112 p.

EURIDES, D.; BENTO, L.R.; SILVA, C.R.; DALECK, C.R. Implantation of the autologous tendon from the superficial flexor of the fingers muscle in the deviation repair of bovine penis. Braz. J. Vet. Res. An. Sci., v. 44, n. 6, p. 415-421, 2007.

EURIDES, D; SILVA, L.A.F.; DALECK, C.R.; FIORAVANTI, M.C.S; GONÇALVES, G.F.; SILVA, O.C.; NADRI, A.B. Implante de cartilagem auricular autóloga no reparo de desvio de pênis de bovinos. Pesq. Vet. Bras., v. 29, n. 3, p. 258-262, 2009. observaram presença de resposta imune intensa, uma vez que foram utilizados materiais não imunogênicos. Neste experimento aos 60 dias de PO, observou-se ausência de sinais clínicos de reação inflamatória local crônica e sistêmica de resposta imunológica de quadro agudo. Uma vez que materiais biológicos perdem a capacidade de gerar reação antigênica após 60 dias de preservação na glicerina a 98\% (Costa Neto, 2000).

\section{Conclusão}

O implante de ligamento nucal alógeno conservado em glicerina a $98 \%$, implantado sobre a túnica albugínea, promove a correção do desvio ventral e lateral direito de pênis em bovinos, sem alterar o comportamento sexual.

GUIMARÃES, G.C.; SCAVONE, A.R.F.; MACHADO, M.R.F.; CRUZ, C. da; CAPALBO, A.C.; SANTOS, A.L.Q. Avaliação histológica de membranas biológicas bovinas conservadas em glicerina e a fresco. Biosci. J., v. 23, n. 3, p.120-127, 2007.

HAFEZ, E.S.E. Reprodução animal. 7. ed. São Paulo: Manole, 2004. 513 p.

MIHURA, H.; CAMPERO, C.M. Lesiones genitales e locomotoras em 5381 toros de carne detectadas clinicamente y por la prueba de capacidad de servicio. Rev. Arg. Prod. Anim., v.15, fascículo 3-4, p. 748-751, 1995.

MOBINI, S.; WALKER, D.F.; CRAWLEY, R.R. An experimental evaluation of the response of the bull penis to carbon fibigir implants. Cornell Law. Rev., v. 72, p. 350-360, 1982.

MOBINI, S.; WALKER, D.F. Desviations of the penis in the bull. Compend. Contin. Educ. Pract. Vet., v. 5, n. 5, p. 280-284, 1983.

RABELO, R.E. Desvio de pênis em bovinos: aspectos epidemiológicos, morfológicos tratamento cirúrgico empregando biomateriais. 2009. 86 f. Dissertação (Doutorado em Ciência Animal) - Escola de Veterinária. Universidade Federal de Goiás, 2009.

SCHALLER, O. Nomenclatura anatômica veterinária ilustrada. São Paulo: Manole. 1999, 614 p.

STEFFEN, D. Genetic causes of bull infertility. Vet. Clin. North Am. Food Anim. Pract., v.13, n. 2, p. 251, 1997.

VAN CAMP, S.D. Bull infertility. Vet. Clin. North Am. Food Anim. Pract., v.13, n. 2, p. 203-231, 1997.

WALKER, D.F. Penile surgery in the bovine: part. III. Med. Vet. Pract., v. 61, n.1, p. 69-71, 1980. 